\title{
Hematopoietic stem cells: ex-vivo expansion and therapeutic potential for myocardial ischemia
}

This article was published in the following Dove Press journal:

Stem Cells and Cloning:Advances and Applications

27 March 2010

Number of times this article has been viewed

Jingwei Lu

Vincent J Pompili

Hiranmoy Das

Cardiovascular Stem Cell Research Laboratory, The Dorothy M Davis

Heart and Lung Research Institute,

The Ohio State University Medical

Center, Columbus, OH 43210 , USA
Correspondence: Hiranmoy Das Cardiovascular Stem Cell Research Laboratories, The Dorothy M Davis Heart and Lung Research Institute, The Ohio State University Medical Center, 460 W. I2th Avenue, BRT 382, Columbus, Ohio 43210 USA

$\mathrm{Tel}+\mid$ 6| 6 688-87| I

Fax + I 614 293-56I4

Email hiranmoy.das@osumc.edu
Abstract: Despite recent advances in cardiovascular medicine, ischemic heart disease remains the major cause of death in the United States and abroad. Cell-based therapy for degenerative diseases like myocardial ischemia using stem cells is currently under serious investigation. Various types of stem cells are being considered to be candidates for cell transplantation in cell-based therapy. Hematopoietic stem cells are one of the most promising cell types as several studies demonstrated their ability to improve ischemic cardiac functions by enhancing neovascularization and by reducing the total size of scar tissue. However, in order to procure sufficient numbers of functional stem cells, ex-vivo expansion technology became critically important. In this review, we focus on the state-of-the-art ex-vivo technology for the expansion of hematopoietic stem cells, and the underlying mechanisms regulating stem cell self-renewal as well as differentiation.

Keywords: ischemic heart disease, ex-vivo expansion, hematopoietic stem cells, cytokines, nanofibers

\section{Introduction}

Ischemic heart disease (IHD, coronary heart disease) causes approximately 1 in every 5 deaths in the United States. It was estimated that nearly 17 million American adults suffered from IHD in 2006. ${ }^{1}$ Myocardial ischemia (MI) is generally caused by the occlusion of a coronary artery due to the deposition of fat, which results in the shortage of oxygen and nutrition. If, left untreated, this will lead to massive cell death, ischemia, and eventually will result in heart failure. The loss of cardiomyocytes and other surrounding cells in the heart tissue causes permanent damage to the patient's heart. Conventional therapies hardly address this issue, and stem cell transplantation is considered to be the only way, which targets the fundamental problem of cell loss. ${ }^{2}$ Administration of progenitor cells from bone marrow was reported to significantly improve the recovery of left ventricular contractile function. ${ }^{3,4}$ Various mechanisms, such as paracrine effect, angiogenesis and cell fusion were found to be important in functional improvement of the heart after stem cell transplantation. However, an insufficient number of biologically functional autologous or allogeneic isolated stem cells, cell viability, and inefficient expansion techniques limit its clinical application. Therefore it is very important to address the critical issues involved in the development of the technology, which will support an efficient and practical ex-vivo stem cell expansion. This technology is important to maintain the expanded stem cell's potential for engraftment, differentiation and long-term sustainability. Different approaches have been developed to achieve large-scale expansion including co-culturing with 
feeder cells, gene transduction and usage of biomaterial scaffolds. In this review, rather than elaborating on the end results of ex-vivo expansion, we will focus on the underlying mechanisms and principles, which modulate stem cell fate. Special emphasis is given to hematopoietic stem cells as they are relatively safe and easy to procure, compared to other sources, and have been demonstrated to posses beneficial effects on the functional recovery of the heart, as reported by various clinical trials. ${ }^{3,4}$

\section{Stem cells for cardiac therapy}

Different types of stem cells have been considered as candidates for cardiac transplantation therapies. Some of the widely studied cells are: skeletal myoblasts; mesenchymal stem cells (MSCs); endogenous cardiac stem cells; and bonemarrow-derived hematopoietic stem cells (HSCs). ${ }^{2}$

\section{Skeletal myoblasts}

Skeletal myoblasts are progenitor cells of skeletal muscle cells and have been used previously as a source of stem cells for the regeneration of ischemic heart tissues. Cardiac muscle cells (or cardiomyocytes) are akin to skeletal muscle in their structure, which brings hope to the investigators that skeletal myoblasts may transdifferentiate into cardiomyocytes. Various animal models with myocardial ischemia have shown beneficial effects after local myoblast transplantation. However, myoblasts remain committed to skeletal muscle fate and do not transdifferentiate into cardiomyocytes after transplantation. Mature skeletal muscle cells do not electromechanically couple with each other and thus do not beat in synchrony with the surrounding myocardium. The mechanism for the improvement of this function is currently under investigation. However, some reports indicate that patients develop arrhythmias after transplantation, which brings into doubt the long-term benefit of myoblasts transplantation. ${ }^{2,5}$

\section{Mesenchymal stem cells}

Bone marrow derived MSCs were shown to be able to transdifferentiate into cardiomyocytes in vitro. This result provided the possibility of using MSCs for heart regeneration therapy. ${ }^{6}$ However, further studies showed that immortalization plays an important role in the transdifferentiation process and MSCs cannot differentiate into functional cardiomyocytes without immortalization. ${ }^{7}$ Some reports showed that direct injection of MSCs into the heart improved ventricular function in ischemic animal models. ${ }^{8}{ }^{8}$ However, since MSCs express many common surface markers similar to cancer cells, the long-term safety of MSCs transplantation needs to be carefully examined. Indeed, reports have shown that MSCs can promote cell growth in vivo and aged MSCs may develop into fibrosarcomas in mice. ${ }^{10}$

\section{Endogenous cardiac stem cells}

A specialized type of cells was isolated from mammalian myocardium and was suggested to be cardiac progenitor cells. Surface markers like c-kit, Sca-1 and Isl-1 were used to isolate these cardiac progenitor cells. In vitro experiments demonstrated the potential of these cells to differentiate into cardiomyocytes, endothelial cells and smooth muscle cells. ${ }^{11-14}$ In vivo transplantation of these cells resulted in the apparent enhancement of cardiac function, ${ }^{15}$ which makes these cells attractive candidates for transplantation. Even though relatively new, endogenous cardiac stem cells have attracted lots of attention. More work need to be undertaken to further characterize these progenitor cells and methods for the large-scale ex-vivo expansion of these cells need to be developed.

\section{Hematopoietic stem cells}

Hematopoietic stem cell transplantation was reported to improve functional cardiac recovery in animal models. ${ }^{16}$ Various mechanisms have been proposed that may be involved in the enhancement of cardiac function. Neovascularization may directly result from the differentiation of HSCs and enhanced by the cytokines secreted by transplanted cells. ${ }^{17}$ Cell fusion between HSCs and cardiomyocytes has also been observed after transplantation which may imply such enhancement may result from this fusion. However, whether these fused cells can provide the population of progenitor-derived cardiomyocytes required for this therapy is still debatable. ${ }^{18}$ However, it seems that even if HSCs can transdifferentiate into cardiomyocytes in vivo, the effect might be minor. It is also possible that rather than directly transdifferentiating into cardiomyocytes, transplanted cells may enhance the proliferation of endogenous progenitors in the heart tissue by a paracrine effect. ${ }^{16,19,20}$

Many groups have carried out clinical trials using bone marrow- (BM) derived HSCs for cardiac repair. Improvements were observed in patients with acute myocardial infarction and those with chronic heart failure due to prior myocardial infarctions, with no serious adverse effects observed (Table 1 ). ${ }^{3,4,21-25}$ These studies suggest that bone marrow transplantation for cardiac repair is both feasible and safe. However the mechanism for this improvement needs to be explored in more detail. Because of the limited number of HSCs available for autologous transplantation, most of the clinical trials use bone marrow rather than a pure population of stem cells. Cardiac 
Table I Clinical trials using bone marrow derived cells

\begin{tabular}{|c|c|c|c|}
\hline Heart diseases & Number of patients & Delivery techniques & Functional improvements \\
\hline Acute myocardial infarction ${ }^{21}$ & 10 & Intracoronary & $\begin{array}{l}\text { Infarct region } \downarrow \\
\text { Infarction wall movement velocity } \uparrow\end{array}$ \\
\hline Acute myocardial infarction ${ }^{23}$ & 30 & Intracoronary & $\begin{array}{l}\text { Global left venticular ejection fraction (LVEF) } \uparrow \\
\text { Left-venticular systolic function } \uparrow\end{array}$ \\
\hline Acute myocardial infarction ${ }^{24}$ & 20 & Intracoronary & $\begin{array}{l}\text { End-systolic volume } \downarrow \\
\text { Regional and global LVEF } \uparrow \\
\text { Thickness of the infarcted wall } \uparrow\end{array}$ \\
\hline Acute myocardial infarction ${ }^{4}$ & 204 & Intracoronary & $\begin{array}{l}\text { Improvement in the global LVEF } \uparrow \\
\text { Clinical end point of death (I year) } \downarrow \\
\text { Recurrence of myocardial Infarction (I year) } \downarrow\end{array}$ \\
\hline Chronic coronary ${ }^{3}$ & 75 & Intracoronary & $\begin{array}{l}\operatorname{LVEF} \uparrow \\
\text { Regional contractility } \uparrow\end{array}$ \\
\hline Chronic coronary ${ }^{25}$ & 10 & Endocardial injection & $\operatorname{LVEF} \uparrow$ \\
\hline
\end{tabular}

repair using relatively pure HSCs may help our understanding of a clearer role of stem cells in the recovery process of heart disease. The long-term effect of transplantation also needs to be monitored. Autologous bone marrow, collected from aged patients, may result in reduced efficiency of transplantation as well as cardiac repair. For such patients, using HSCs derived from cord blood may provide an alternative option. It is yet to be established what the optimum conditions are for successful transplantation and cardiac repair.

Till now, HSCs seems to be one of the best-characterized cells for cardiac therapy related to ischemic diseases. However, limited cell numbers available for autologous transplantation severely hinders the regenerative capability post-transplantation. The ex-vivo expansion of HSCs from bone marrow, or other sources such as those from human umbilical cord blood, seem to be a critical step before transplantation. Extensive work has been undertaken to explore the mechanism that control the HSCs self-renewal process in vitro as well as in vivo.

\section{Signaling pathways governing HSC fate}

It is well known that cultured in vitro stem cells, especially HSCs, tend to differentiate rather than self-renew. Several factors influence the fate of HSCs in self-renewal versus differentiation in vivo, and three signaling pathways have been found to be important in this process ie: Notch pathway; Wnt pathway; and the Smad pathway (Figure 1). ${ }^{26}$

\section{Notch signaling}

Notch proteins are involved with microenvironment intercellular cell-fate decisions such as cell survival, self-renewal for stem cells and lineage-determination for developing cells. Notch signaling in high or low levels are lethal at the embryonic level. The Notch signaling network is composed of a family of four Notch receptors (Notch 1, Notch 2, Notch 3, Notch 4) and five ligands from the Jagged (Jagged-1 and Jagged-2) and Delta (Delta-like-1, Delta-like-3 and Deltalike 4) families, plus modifier proteins from the Fringe family (Lunatic, Manic, and Radical Fringe). ${ }^{27}$ Notch signaling receptors have three domains: extra cellular; transmembrane; and intracellular domains. Upon activation, intracellular domains of Notch receptors undergo cleavage and then enter the nucleus regulating variety of genes. Studies have shown that Notch, Delta and Jagged ligands can enhance the expansion of hematopoietic progenitors in vitro. ${ }^{28,29}$ Activation of endogenous Notch signaling with immobilized Delta-1 in human $\mathrm{CD}^{3} 4^{+} \mathrm{CD} 38$ - cord blood precursors induced a 100 -fold increase of CD34 ${ }^{+}$cells compared to controls, ${ }^{29}$ even though high a concentration of Delta 1 results in apoptosis of CD $34^{+}$precursors. ${ }^{30}$ Immobilized Delta and Jagged ligands may act through the activation of HES-1 genes, which encodes a basic helix-loop-helix transcription factor and lies downstream in the Notch signaling pathway. Transduction with HES-1 can preserve the long-term reconstituting activity of CD $34^{\text {low/- }} \mathrm{c}-\mathrm{Kit}^{+} \mathrm{Sca}-1^{+} \mathrm{Lin}^{-}\left(34^{-} \mathrm{KSL}\right)$ in vitro. ${ }^{31}$ In vivo, osteoblasts may act as a source of Jagged-1, which indicates that osteoblasts serve as an important part of HSCs niche. ${ }^{32}$

\section{Wnt signaling}

Wnt signaling plays an important role in regulating cell fate in a variety of tissues, and is required for dorso-ventral axis specification during early development. Wnt signaling can activate the canonical $\beta$-catenin dependent pathway and two non-canonical pathways. During the activation of the canonical pathway, Wnt ligands bind to cell-surface 


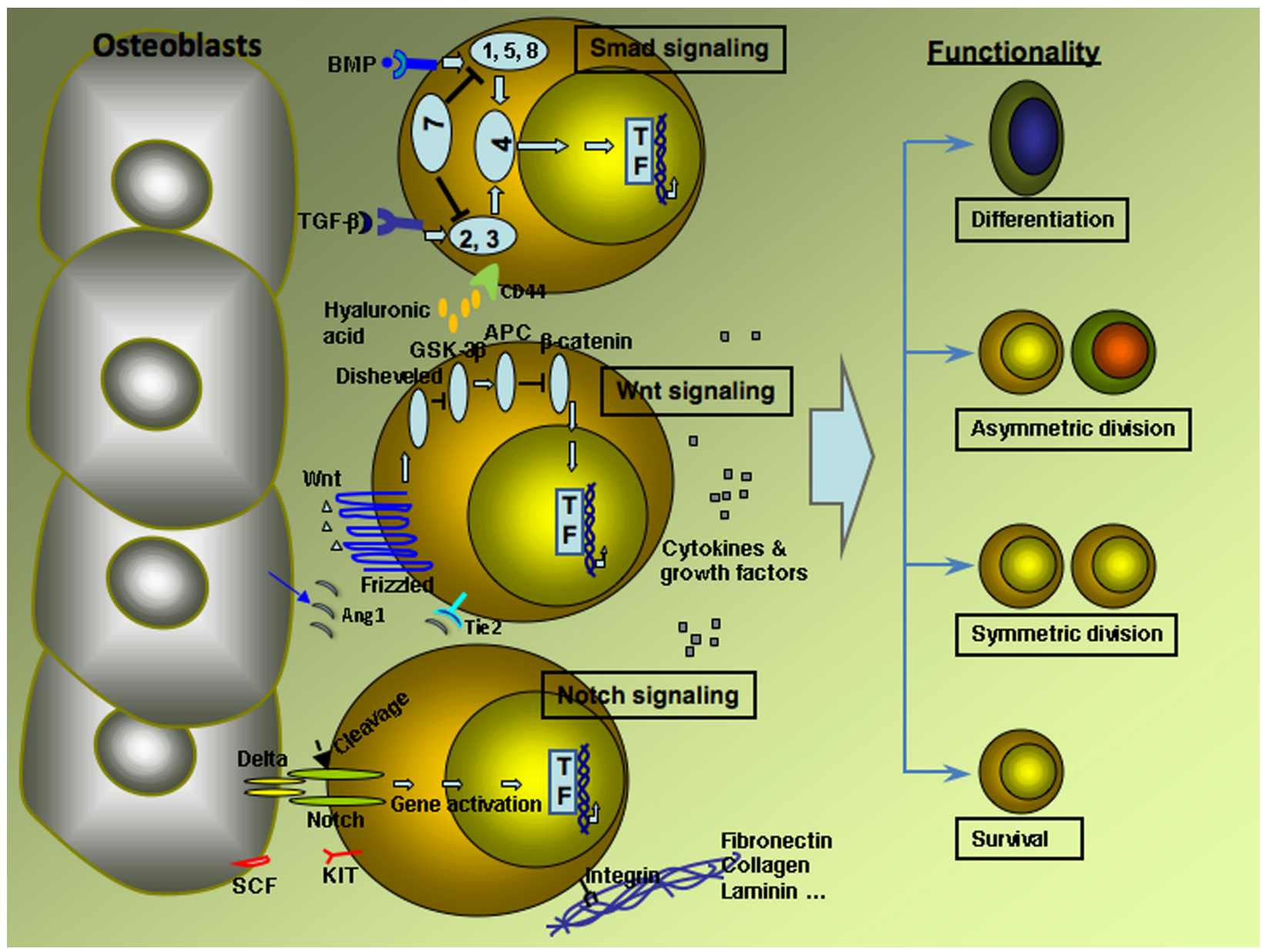

Figure I Major signaling pathways and factors influence HSCs fate in vivo.

Notes: The Smad signaling: BMP or TGF- $\beta$ binds to surface receptors expressed on HSCs, which activate receptor regulated Smad (R-Smad), Smad I, 5, 8 or Smad 2, 3 respectively. Activated R-Smad binds to Smad 4 and forms a complex, which enters to the nucleus and activate variety of genes.

The canonical Wnt signaling:Wnt binds to surface receptors of the Frizzled family protein expressed on HSCs. Then the receptors activate Disheveled family protein, which in turn inhibit glycogen synthase kinase (GSK-3) and adenomatosis polyposis coli (APC). This inhibition stabilizes $\beta$-catenin and leads to the activation of transcription factors through $\beta$-catenin. Notch signaling: the extracellular domain of Notch signaling receptor binds to Jagged or Delta proteins expressed on osteoblasts. Upon binding, intracellular domain of Notch receptor undergoes cleavage and enters to the nucleus, which regulates a variety of genes. Other factors that influence the fate decision of HSCs include cytokines and growth factors, integrin binding to extracellular matrix molecules, SCF, AngI, CD44 etc, which function as either enhancing the survival ability of HSCs or mediating cell division or inducing differentiation.

receptors of the frizzled family, which in turn activate disheveled family proteins with results in the stabilization of $\beta$-catenin. $\beta$-catenin translocates into the nucleus and activates gene transcription. Expression of Wnt-5a and Wnt$10 \mathrm{~b}$ were found in yolk sac and fetal liver, which were the sites for fetal hematopoiesis. Wnt-5a and Wnt-10b can also stimulate HSCs expansion in vitro. ${ }^{33}$ It was hypothesized that Wnt's function lay in stabilizing and translocating $\beta$-catenin into nuclear following canonical Wnt signaling pathway. $\beta$-catenin/T cell factor complex then acts as a transcriptional regulator for different target genes, which in turn enhance the self-renewal of HSCs. Indeed, overexpression of activated $\beta$-catenin leads to a $10^{2}$ to $10^{3}$ fold expansion of the pool of HSCs in long-term cultures. Activation of Wnt also induces an increased expression of HoxB4 and Notch1 genes which can also promote HSCs expansion. ${ }^{26,34}$

\section{Smad signaling}

The Smad signaling pathway functions downstream of transforming growth factor beta (TGF- $\beta$ ) and bone morphogenetic proteins (BMPs). Blockage of the entire Smad pathway by overexpression the inhibitory Smad7 can promote the self-renewal capacity of HSCs without disturbing the differentiation capacity. ${ }^{35}$ This is consistent with the inhibitory role of TGF- $\beta$, which is illustrated by release of HSCs from quiescence using antisense TGF- $\beta$ stimulus. ${ }^{36}$ BMPs are suggested to play a critical role in the specification of hematopoietic tissue from the mesodermal 
germ layer. The effect of BMPs on adult $\mathrm{CD} 34^{+}$cells act in a dose dependent manner. While low concentration of BMP-4 induced proliferation and differentiation of $\mathrm{CD} 34^{+}$ CD38-Lin ${ }^{-}$cells, high concentrations of BMP-4 can help to maintain the repopulation capacity. ${ }^{37}$ It has also been shown that sonic hedgehog (Shh) genes induce the expansion of human HSCs through the modulation of the BMP pathway. ${ }^{38}$ It seems that the action of Smad is context dependant, and sometimes leads to different results in vitro and in vivo. The dose depended effect of BMPs on HSCs may indicate the involvement of other signaling cascades.

\section{Stem cell micro-environment}

The stem cell microenvironment, called niche, has a strong impact on the fate of stem cells in vivo. In the niche, stem cells can either undergo asymmetric division, giving rise to one daughter cell, which is further differentiated into certain lineage, or symmetric divisions; which results in two new stem cells depending on the signal from its environment. Two basic components constitute the stem cell niche: niche cells and the extra-cellular matrix (ECM). Niche cells send signals to stem cells, which control their quiescence, proliferation or differentiation. ECM provides physical and chemical support for stem cell function. The ECM is also being constantly modified by both niche cells and stem cells, which in turn modulate stem cell behavior. In some tissues there is only one or a few stem cell types for each niche, like hematopoietic stem cells, satellite muscle cells and intestinal epithelium, while in other tissues there are many stem cells per niche, like central nervous system SVZ stem cells and epithelial stem cells of the hair follicle bulge. ${ }^{39}$

HSCs are one of the most well studied somatic stem cells and the niche is relatively well-characterized. ${ }^{40}$ Single HSCs can result in the long-term reconstitution of the lymphohematopoietic system, which shows its strong capacity for expansion in vivo. ${ }^{41}$ The HSC niche is populated by a variety of cells (osteoblasts, osteoclasts, endothelial cells, fibroblasts, and macrophages), ECM molecules and cytokines. Many factors have been demonstrated to influence HSCs maintenance in vivo and recently there is new evidence demonstrating that cytokines play an important role in regulating HSCs differentiation. ${ }^{42}$

\section{Niche cells}

Even though different cell types populate the HSCs niche, it is believed that osteoblasts are one of the most crucial cells supporting HSCs maintenance, and together with osteoclasts are the two major bone remodeling cells. Osteoblasts secrete many cytokines such as: granulocyte-colony-stimulating factor (G-CSF); macrophage colony stimulating factor (MCSF); granulocyte macrophage colony stimulating factor (GM-CSF); Interleukins (IL)-1, IL-6, IL-7; osteoprotegerin $(\mathrm{OPG})$; receptor activator of nuclear factor kappa B; tumor necrosis factor (TNF)- $\alpha$; and vascular endothelial growth factor (VEGF), all of which can modulate stem cell function. ${ }^{43}$ Osteoblasts produce stromal cell-derived factor (SDF)-1, which is a chemo-attractant recognized by the CXC chemokine receptor 4 (CXCR4) of HSCs. ${ }^{44}$ Lack of bone marrow seeding was seen in SDF-1 deficient murine embryos. ${ }^{45}$ SDF-1 can also stimulate the growth and survival of $\mathrm{CD} 34^{+}$progenitor cells, possibly through the phosphoinositol 3-kinase $(\mathrm{PI} 3 \mathrm{~K})$ /protein kinase $(\mathrm{PKB})(\mathrm{Akt})$ pathway and mitogen-activated protein kinase (MAPK)/p90 ribosomal S6 kinase pathway. ${ }^{46-48}$

It seems that cell adhesion play a very important role in the interaction between osteoblasts and HSCs. Many adhesion molecules expressed on osteoblasts and HSCs can mediate HSCs-osteoblasts adhesion, eg, inter-cellular adhesion molecule-1 (ICAM-1); lymphocyte function associated antigen-1 (LFA-1); vascular cell adhesion molecule-1 (VCAM-1); very late antigen-4 (VLA-4); and N-cadherin. Osteoblasts express angiopoietin-1 (Ang-1), which in turn activates its receptor, Tie 2 and promotes the tight adhesion of stem cells to their niche. It is proposed that this adhesion results in HSCs quiescence and survival. ${ }^{49}$ However, specific roles of these adhesion molecules are still not fully understood.

The BMP signaling pathway is an inducer of the hematopoietic system, and has a very important role in regulating adult HSC development. Long term, HSCs attach to $\mathrm{N}$-cadherin ${ }^{+} \mathrm{CD}_{4} 5^{-}$osteoblastic (SNO) cells through $\mathrm{N}$-cadherin and $\beta$-catenin junction molecules. Conditional inactivation of BMP receptor type IA leads to an increase in SNO cells, which in turn induces a proportional increase of HSCs. This suggests that adhesion molecules might provide an important signal for HSCs maintenance of pluripotency. ${ }^{50,51}$

The important role of osteoblasts to HSCs is also demonstrated through the Notch signaling pathway. Activation of osteoblast-specific parathyroid hormone (PTH) or PTH related protein (PTHrP) receptors leads to a higher level of Notch ligands and Jagged 1 in osteoblasts; and an increase in HSCs with Notch1 activation. Blocking Notch cleavage using $\gamma$-secretase inhibitor can reduce the supportive effect back to a base level. This indicates that osteoblasts can activate the HSCs through the Notch signaling pathway, which in turn results in an expansion of HSCs in vivo. ${ }^{32}$ 
Overall, osteoblasts can regulate HSCs through different ways. It seems that cell adhesion is more important for homing and survival, while other factors favor regulating the maintenance of the HSC pool. Other cell types can also regulate HSCs through various mechanisms. For example, fibroblasts are one of the main sources of extra-cellular matrix molecules, which is important in supporting HSC function. However, these cell types are less well studied and are thought not to be as important as osteoblasts.

\section{Extra-cellular matrix}

More and more evidence has been demonstrated that the extra-cellular matrix (ECM) provides a profound role in regulating cell fate. The ECM can regulate stem cell activity through: transmission of adhesion signals; control cell geometry; mechanical property; and nanotopography.

\section{Stem cell-ECM interactions through integrins}

Integrins are important mediators of cell adhesion and play a critical role in stem cell homeostasis. $\beta_{1}$ integrin deficient embryos show an impaired migration of hematopoietic stem cells in the early developmental stage. ${ }^{52} \beta_{1}$ integrins expressed by HSCs are in a low-affinity state, which require transactivation by agonists to induce a high affinity ligand binding state. This activation has been shown to occur for the $\beta_{1}$ integrins VLA-4, and VLA-5; receptors for both fibronectin (VLA-4 and VLA-5) and the stromal cell surface protein VCAM-1(VLA-4 only). ${ }^{53}$ Fibronectin contains connecting segment-1 (CS-1) and an arginyl-glycyl-aspartic acid (RGD) motif, which binds to VLA-4 and VLA-5 respectively. Both segments are important for growth support and proliferation of hHSCs. ${ }^{54}$ It is suggested that adhesion of HSCs to fibronectin may trigger signals related to the cytoskeleton pathway, which in turn activate signals that enhance both cell survival and growth. ${ }^{53}$

\section{Stem cell-ECM interaction through physical properties}

It is well known that myocardial development is potentially regulated by cell shape, and the differentiation of capillary endothelial cells is partially regulated by insoluble ECM molecules. ${ }^{55}$ However little is known about the influence of the physical property of the ECM on adult stem cell fate. Most of the current knowledge of such physical property influences come from studies using MSC and other connective tissues. However, it has been shown that the nanoscale structure also has profound a influence on HSCs ex-vivo expansion. ${ }^{56}$
Mechanical signals of the ECM can induce a changes in cytoskeletal tension and mechanosensitive or osmosensitive ion channels, and regulate cell fate from seconds to hours. Cell shape can regulate RhoA, a guanosine-5'-triphosphatease (GTPase) protein, activity in multiple ways. For MSCs, cell shape restriction can switch the lineage commitment by the RhoA/Rho kinase (ROCK) pathway. Constitutive activation of ROCK induced osteogenesis while dominant negative expression of RhoA leads MSCs to become adipocytes. ${ }^{57}$

The stiffness of the ECM can influence cell migration, apoptosis, proliferation and differentiation. ${ }^{58}$ It has been shown that MSCs commit to different phenotypes according to the stiffness of the matrices, which mimic the elasticity of different cell types. Soft matrices that mimic brain tissues are neurogenic, stiffer matrices that mimic muscle tissues are myogenic, whereas rigid matrices mimic collagenous tissues are osteogenic..$^{59}$ Using different stiffness of polyacrylamide gels, Jessamine Winer and colleagues have shown that if cultured in $250 \mathrm{~Pa}$ gels, (which mimics the elasticity of bone marrow), MSCs become quiescent. Adhesion to a stiff substrate can bring the quiescent cells back into cell cycle again. ${ }^{60}$ Neural stem cells (NSC) also respond differently on materials with a different modulus. On materials with a modulus of 1,000-10,000 Pa, NSCs differentiate into glial cells. Lowering the stiffness to $10 \mathrm{~Pa}$ will totally inhibit NSCs self-renewal and differentiation, whereas in between, certain neural specific markers are expressed. ${ }^{61}$ These experiments provide evidence that biomaterials mimicking the local in vivo environment of stem cells may provide a better support for in vitro culturing and expansion.

The structural changes of the ECM, which regulate cell shape, ranges from macro to nano scale. These influences include surface topography, surface roughness, fiber diameter and other factors. Neural stem cells grown on polyethersulfone (PES) fiber meshes with fiber diameters of $283 \mathrm{~nm}, 746 \mathrm{~nm}$ and $1452 \mathrm{~nm}$ show a different pattern towards differentiation. Cells were shown to be stretched multi-directionally on 283-nm fibers and grow along a single fiber axis on larger fibers. The NSCs show a higher degree of proliferation and cell spreading on PES fiber mesh as the fiber diameter decreases, if cultured with $20 \mathrm{ng} / \mathrm{mL}$ fibroblast growth factor (FGF)-2. ${ }^{62}$ Human MSCs cultured on $350 \mathrm{~nm}$ width nanograting show an alignment and elongation of their cytoskeleton and nuclei along the nanograting. Certain neuronal markers were up regulated such as microtubule-associated protein 2 (MAP-2). When compared with retinoic acid, the nanotopographic cues show a stronger effect for neurogenesis. ${ }^{63}$ 
The mechanisms of the influence of nanotopography on stem cells is not yet fully understood. Several reports have been published searching for pathways that may be involved in cell response to biomaterial surface changes. Arnold and colleagues demonstrated the relationship between the spacing of the nano-structure and integrin clustering. Gold nanodots coated with integrin binding RGDfK peptide was positioned with $28,58,73$, and $85 \mathrm{~nm}$ spacing at the interface. Limited cell attachment and reduced focal adhesion and actin-stress fibers of the cells were shown with a separation of larger than $73 \mathrm{~nm}$ spacing, while a separation of $\leq 58 \mathrm{~nm}$ between the dots allowed effective adhesion. These results indicate that the spacing of the peptide may influence the cluster formation of integrins, which then leads to regulation of the cell properties through cytoskeleton related pathway. ${ }^{64}$ However, most of the reports till now are signaling pathway pre-selected. Recently, a number of reports have demonstrated a global analysis of the influence of nanotopographical structures on osteogenesis using differential proteomics when human bone marrow osteoprogenitors were used. Many molecules related to the Erk1/2 pathways were upregulated and are purported to play a role in the regulation of progenitors' differentiation induced by nano-geometry. ${ }^{65}$

\section{Ex-vivo expansion of hematopoietic stem cells}

Even though significant efforts have been given to the ex-vivo expansion of hematopoietic stem cells and many mechanisms controlling the fate of HSCs have been revealed, we are still far from a full understanding. In order to achieve an ex-vivo expansion, suitable for transplantation, the following two requirements need to be fulfilled:

1. The large scale expansion of HSCs without compromising the pluripotency and long-term repopulation capacity (self-renewal).

2. Such expansion should provide safe and transplantable HSCs. This requires the expansion to be feeder cell-free, serum proteins or microbial agents free.

Exposure of human tissues to xenogenic products can raise the risk of contamination by infectious agents. Studies show that human embryonic stem cells (hESCs) cultured with animal-derived serum replacements on mouse feeder layers can take up non-human sialic acid Neu5Gc from the culture medium. Transplantation using these hESCs might be compromised; as most adults have circulating antibodies against Neu5Gc. ${ }^{66}$ Genetically modified HSCs for expansion will also introduce possible clinical complications and therefore is not preferred for ex-vivo expansion.
Different factors can influence the result of ex-vivo expansion such as: initial cell concentration; duration of culture; and the purity of stem cells. Current expansion studies focus on two factors: cytokines and the physical culture environment for the cells. ${ }^{67}$ Chromatin modification has also been revealed to play a role in HSCs self-renewal and differentiation in vitro. ${ }^{68}$ Recent advances have indicated that chromatin-modifying agents, 5-aza-2'-deoxycytidine (5 azaD) and trichostatin A (TSA) can help to retain the repopulation capacity of HSCs in addition to cytokines while expanding in vitro. ${ }^{69}$

\section{Cytokines in ex-vivo expansion}

Historically it was shown that murine HSCs can be amplified in vitro in stromal-based long-term culture. ${ }^{70}$ Miller and Eaves reported a three-fold net increase in long-term lymphomyeloid repopulating cells following culture in serum-free media with interleukin-11, Ftl3-ligand and steel factors (SF) without impairing their in vivo regenerative potential. This demonstrated the possibility to expand HSCs in vitro without using feeder cells. ${ }^{71}$

What is the upper limit of HSCs self-renewal? Long-term reconstituting cells (LTRCs) have been monitored during sequential transplantations. After four transfers, LTRCs gain a total 8400-fold expansion without any decline of expansion capability. What's more intriguing is that expansion can be enhanced in vivo by administration of SCF and IL- $1 .^{72}$ This demonstrated that not only these cells have the ability for high fold-expansion but also this potential can be regulated by extrinsic factors. Indeed, by using a limited dilution method, Ueda and colleagues reported that human HSCs can expand by a factor of 4.2 when cultured in serum free conditions with the combination of Flk2/Flt3 ligand (FL), stem cell factor (SCF), thrombopoietin (TPO) and IL-6 and soluble IL-6 receptor. ${ }^{73}$

Two problems with HSCs in vitro expansion are the shortterm culture period and low overall expansion efficiency. Even though short-term expansion of HSCs was shown to be possible, long-term expansion usually results in differentiation and reduced engrafting potential. For example, human cells capable of repopulating non-obese diabetic (NOD)/severe combined immunodeficiency (SCID) mice (SCID-repopulating cells; SRC) were able to be maintained in serum-free condition and showed a 2.4-fold increase after 4 days of culture. However, all the SRCs were lost after 9 days of culture. ${ }^{74}$

The cause of this low efficient expansion was studied using $\mathrm{CD}_{3} 4^{+}$cord blood-derived cells cultured with 
IL-3, IL-6, granulocyte colony stimulating factor, SF and Flt3-ligand. During short time culturing, the majority of the culture initiating cells and in vivo lympho-myeloid competitive repopulating units have divided at least once. This means that during short-term culturing, the HSCs were able to respond to the cytokines and enter the cell cycle. The limited in vitro HSCs expansion may be due to asymmetrical cell division and inadequate long term HSCs survivability. ${ }^{75}$ In fact, HSCs cultured in medium without any cytokines and other additional factors may undergo massive apoptosis within several days. Thus it would be necessary to search for cytokines and other factors that can potentially influence HSC survival and cell fate decisions. It was reported that TPO and SCF could efficiently promote the viability of a subpopulation of Lin-Sca-1+ bone marrow progenitors. Even though within 40 hours cells treated with SCF showed higher viability; the long-term viability-promoting effect of TPO was similar to that of SCF. ${ }^{76}$ The possibility of cytokines influencing cell fate decisions was also studied. Using a combination of SF, IL3, GM-CSF and erythropoietin, the number of transplantable human lymphomyeloid stem cells was enhanced 10-fold, while the combination of SF, Flt3 and IL-6 gives a 3-fold increase in this population in NOD/SCID immunodeficient mice. Since the regenerating human progenitor populations in such primary mice are known to be maximally cycling, the main effects of growth factors should be on self-renewal decisions rather than proliferation. ${ }^{77}$

Currently, different combinations of growth factors have been used in various expansion experiments together with other factors that may help HSCs expansion, such as a soluble form of hedgehog factor; sonic hedgehog, which may be mediated through modulation of BMP signaling pathways. However, the optimum choice of these factors has not yet been established. The focus of future studies might be put on finding the proper combination of factors, which can promote survival in long-term culturing and also promote the self-renew decisions of HSCs. It is clear now, that the soluble factors alone will not be able to provide satisfactory expansion. Combining other cues, like biomaterials, with cytokines may provide a superior level of viability and thus increase the expansion efficiency.

\section{Biosynthetic materials in ex-vivo expansion}

Even though much effort has been spent on different cytokine-mediated activation of HSC proliferation, only limited success has been achieved. This may be due to the extreme sensitivity of HSCs to their microenvironment. Multiple factors, such as: fluctuations in cytokine concentrations; oxygen tension; temperature; and cell ECM interactions, can trigger HSC differentiation cascades and lead to the depletion of HSCs in the culture. Great promise has been shown in the use of biomaterials in stem cell ex-vivo expansion following the discovery that $3 \mathrm{D}$ tantalum-coated porous biomaterial was able to support long-term hematopoietic progenitor cell (HPC) culture and expanded HPCs up to1.5-fold in numbers after 1 week, and 6.7-fold increase in colony-forming ability after 6 weeks without any cytokines. ${ }^{78}$ These results indicated that biomaterials might be able to enhance the long-term survivability of HSCs even without the aid of added cytokines. Various materials without modification have been tested on their ability to support HPC expansion in a serum-free medium. ${ }^{79}$ However, the efficiency of these biomaterials, without the addition of cytokines or modification is relatively low and not suitable for HPC expansion applications.

In order to enhance the ex-vivo expansion efficiency, investigators tried to modify the biomaterials and improve their comparability to the bone marrow niche. Currently research is focusing on two aspects: the interaction between hematopoietic stem-progenitor cells (HSPCs) and nanosegments, and the topology of the biomaterials. Many ECM molecules have been tested on their ability to support cell adhesion conjugated with biomaterials. Thin films of polyethylene-alt-maleic anhydride (PEMA) were coated with various components eg, fibronectin; heparin; heparan sulfate; hyaluronic acid; tropocollagen I; and co-fibrils of collagen I with heparin or hyaluronic acid. Adhesion areas of individual cells have been assessed, and fibronectin showed the strongest adhesion of HSPCs, which is mediated by $\alpha_{5} \beta_{1}$ integrin. Surfaces modified by heparin resulted in much less adhesion area, which is mediated by selectins (CD62L) ${ }^{80}$ In order to directly test whether better adhesion correlated with a better expansion ability of HSPCs, Sagar and colleagues have investigated murine bone marrow cells expansion on fibronectin-coated plate in the presence of cytokines. Coating with fibronectin significantly improved the expansion of primitive hematopoietic stem cells. However, no apparent activation of the cell cycle was observed. Instead an up-regulation of CD29 and VLA-4 was seen as compared to the normal BM cells. This indicated that rather than enhancing the overall level of proliferation, fibronectin specifically increased the ability of primitive HSCs maintenance. ${ }^{81}$ Poly-ethylene terephthalate (PET) films coated with CS-1 motifs and RGD motif were also used to enhance the proliferation of HSCs. After 10 days culture of $\mathrm{CD} 34^{+}$ cells in a serum-free medium, in combination with human 
thrombopoietin, SCF, flt3-ligand and IL-3, the total cell number of cells was increased by approximately 590-fold, total colony forming unit were increased 76-fold and the long-term culture initiating cells were increased by 3 -fold on the CS-1 peptide-modified films, while the PET film coated with the RGD peptide was less efficient. ${ }^{82}$ However, biological functionality, pluripotency and self-renewal of these expanded-cells was not tested. These results showed that covalently immobilized adhesion peptides could replace the direct use of fibronectin and significantly enhance the expansion of HSPCs cultured from umbilical cord blood.

Other modifications have also studied to improve the adhesion between the HSPCs and the biomaterials. A comparison was performed among unmodified, hydroxylated, carboxylated and aminated nanofibers and films using total umbilical cord blood-derived mononuclear cells. It was shown that aminated nanofiber mesh and film are more efficient in supporting the expansion of the CD34+ cells. It seems that the adhesion of HSPCs may play an important role, since cells attached to aminated materials grew much better than the other materials. ${ }^{83}$

The dimension of the materials can also alter the results of the expansion. By using 3-D PET scaffolds conjugated with surface-immobilized fibronectin, $\mathrm{CD} 34^{+}$human umbilical cord blood cells can be expanded 100-fold and long-term culture initiating cells can be expanded 47-fold. The expanded $\mathrm{CD} 34^{+}$cells can successfully reconstitute hematopoiesis in NOD/SCID mice. This significant improvement of the maintenance of $\mathrm{CD} 34^{+}$multipotency and engraftment efficiency may be due to better mimic of the microenvironment for $\mathrm{CD} 4^{+}$maintenance as compared to the $2 \mathrm{D}$-PET film. ${ }^{84} \mathrm{~A}$ more detailed comparison between 3-D scaffolds with 2Dfilm was performed using aminated scaffolds. 3-D aminated nanofiber meshes resulted in a higher cell adhesion and percentage of HSPCs as compared to 2-D film. This better adhesion may play a role in its higher clonogenic abilities. ${ }^{83}$ Other topological factors can also influence the HSPC expansion. For example, the spacer through which the amino groups are conjugated to nanofiber surface, can significantly alter the expansion results. The highest expansion efficiency was observed when using ethylene and butylene spacers, which showed a 200 and 235 -fold expansion of CD $34^{+} \mathrm{CD} 45^{+}$cells respectively. The NOD/SCID mice engraftment potential of HSPCs expanded on these two spacers is also much higher than on aminohexyl conjugated nanofiber. ${ }^{56}$ The shorter spacer may provide certain spacial restrictions on amino group patterning, which may facilitate integrin clustering and enhance the adhesion of the HSCs. These reports showed that the scaffold topography can definitely play a role in HSCs exvivo expansion and optimizations on these topography cues may significantly enhance the expansion efficiency.

In summary, it seems that several things might be important in the construction of biomaterials: a porous 3D structure; the modification of the materials to enhance adhesion of HSCs; and proper spacing of the binding motif or nanofibers. Still the design of suitable biomaterials for stem cell expansion is very delicate work, especially with little guidance. Better biomaterials could be synthesized, as many workers have done in order to determine the underlying principles of HSC interaction with their physical environment.

\section{Preclinical evaluation of expanded HSCs}

Even though tremendous efforts were spent on ex-vivo expansion of HSCs, few reports evaluated the functionality and potential therapeutic efficacy of the expanded HSCs. Using PES nanofiber-scaffolds, which are chemically modified by amination our lab has successfully expanded CD $133^{+} /$ CD $34^{+}$cells from freshly isolated human cord blood. ${ }^{85}$ The therapeutic potential of these cells was evaluated in a myocardial infarction rat model. The ischemic rats transplanted with expanded HSCs showed an elevated heart function and neovascularization. One of the intriguing things is that nanofiber expanded cells are more functionally efficient than that of the freshly isolated cells in both functional recovery and generating neovascularization in the ischemic tissues. ${ }^{17}$ This improved functionality might be partially explained by the higher expression of CXCR4 in nano-fiber expanded cells as compared to the freshly isolated HSCs. ${ }^{85}$ Using different expansion techniques, others have also showed that ex-vivo expanded cells improved the cardiac function after myocardial infarction in murine model. ${ }^{86}$ Numerous clinical trials have been successfully performed worldwide using ex-vivo expanded stem cells for the treatment of hematological malignancies. Expanded stem cells were able to achieve durable engraftment to the hematopoietic system with very few side effects. ${ }^{87}$ The long-term effect of stem cells on IHD is required to be explored, as arrhythmogenicity is one of the potential concerns. Poor cell-cell coupling, incomplete differentiation of engrafted cells or a heterogeneous distribution of action potentials induced by therapeutic cell fusion can generate arrhythmia. Even though no clinical trials using HSCs has shown such effects, the possibility needs to be investigated. ${ }^{88}$ Long-term effects of expanded stem cell transplantation need to be evaluated carefully, as potential graftversus-host disease might also be a cause of concern. As there are no immunocompromised porcine models (large animal) 
available, risk of xenogeneic transfer using expanded stem cells has not been investigated sufficiently. Using immunosuppressive drugs might have side effects on transplanted stem cells. Current research is focusing on optimization of expanded stem cell delivery and the effects on large animal models. Autologous bone marrow cells were used to optimize stem cell delivery methods in an induced acute MI model. ${ }^{89}$ The detailed mechanism of the cardiac repair needs to be clarified in large animal models before translation to the clinic. ${ }^{89,90}$ Genetically modified stem cells may be functionally effective for the therapeutic purposes, however, the long-time safety of such therapy should also be evaluated. ${ }^{91}$ Many other factors have been suggested to influence the outcome of transplantation eg, sex; age; infarct size and location; time from symptom onset to reperfusion; and baseline left ventricular ejection fraction. ${ }^{92}$ Most of the current clinical trials using expanded or non-expanded stem cells are relatively small and not properly controlled. Thus, intermediate to large-size, double blinded and randomized clinical trials seem to be necessary to establish routine stem cell therapy. ${ }^{92}$

\section{Future directions}

As procedural complications involved with the procurement of bone marrow-derived stem cells and that aging and disease state diminishes the functional ability of isolated stem cells, is it possible to use allogeneic cord blood-derived HSCs for future cardiac regenerative therapy? Tremendous efforts have been made on ex-vivo expansion of HSCs isolated from human umbilical cord bloods. However, we still have a long way to go before we reach to our destination. Even after we achieve the desired expansion volume, will the ex-vivo expanded HSCs be suitable for the treatment of IHD? Is HLA-matching enough for allogeneic transfer of expanded stem cells? Which mode will be more appropriate to deliver these stem cells, intravenous or intramyocardial? Can we expand HSCs from frozen cord blood in a sufficient numbers to overcome the limited sources? What will be long-term effects of stem cell therapy for IHD? As ethical issues are not involved, ex vivo isolated stem cells are both easy to procure and manipulate. Even though many questions remain to be answered before its clinical application the adult stem cell research field is growing very fast and, important improvements may be seen in the near future.

\section{Acknowledgements}

This work was supported in part by National Institutes of Health grants, K01 AR054114 (NIAMS), SBIR R44
HL092706-01 (NHLBI), Third Frontier Projects, Ohio Technology, BRCP Grant and The Ohio State University start-up fund for stem cell research.

\section{References}

1. Lloyd-Jones D, Adams R, Carnethon M, et al. Heart disease and stroke statistics - 2009 update: a report from the American Heart Association Statistics Committee and Stroke Statistics Subcommittee. Circulation. 2009;119(3):480-486.

2. Segers VFM, Lee RT. Stem-cell therapy for cardiac disease. Nature. 2008;451(7181):937-942.

3. Assmus B, Honold J, Schachinger V, et al. Transcoronary transplantation of progenitor cells after myocardial infarction. New Engl J Med. 2006;355(12):1222-1232.

4. Schachinger V, Erbs S, Elsasser A, et al. Intracoronary bone marrowderived progenitor cells in acute myocardial infarction. New Engl J Med. 2006;355(12):1210-1221.

5. Laflamme MA, Murry CE. Regenerating the heart. Nat Biotechnol. 2005;23(7):845-856.

6. Makino S, Fukuda K, Miyoshi S, et al. Cardiomyocytes can be generated from marrow stromal cells in vitro. J Clin Invest. 1999;103(5):697-705.

7. Liu Y, Song JA, Liu WX, Wan Y, Chen XC, Hu CJ. Growth and differentiation of rat bone marrow stromal cells: does 5 -azacytidine trigger their cardiomyogenic differentiation? Cardiovasc Res. 2003;58(2):460-468.

8. Ma J, Ge JB, Zhang SH, et al. Time course of myocardial stromal cell-derived factor 1 expression and beneficial effects of intravenously administered bone marrow stem cells in rats with experimental myocardial infarction. Basic Res Cardiol. 2005;100(3):217-223.

9. Shake JG, Gruber PJ, Baumgartner WA, et al. Mesenchymal stem cell implantation in a swine myocardial infarct model: Engraftment and functional effects. Ann Thorac Surg. 2002;73(6):1919-1925.

10. Li H, Fan X, Kovi RC, et al. Spontaneous expression of embryonic factors and $\mathrm{p} 53$ point mutations in aged mesenchymal stem cells: A model of age-related tumorigenesis in mice. Cancer Res. 2007; 67(22):10889-10898.

11. Beltrami AP, Barlucchi L, Torella D, et al. Adult cardiac stem cells are multipotent and support myocardial regeneration. Cell. 2003; 114(6):763-776.

12. Oh H, Bradfute SB, Gallardo TD, et al. Cardiac progenitor cells from adult myocardium: Homing, differentiation, and fusion after infarction. P Natl Acad Sci U S A. 2003;100(21):12313-12318.

13. Cai CL, Liang XQ, Shi YQ, et al. Isl1 identifies a cardiac progenitor population that proliferates prior to differentiation and contributes a majority of cells to the heart. Dev Cell. 2003;5(6):877-889.

14. Bu L, Jiang X, Martin-Puig S, et al. Human ISL1 heart progenitors generate diverse multipotent cardiovascular cell lineages. Nature. 2009; 460(7251):113-117.

15. Dawn B, Stein AB, Urbanek K, et al. Cardiac stem cells delivered intravascularly traverse the vessel barrier, regenerate infarcted myocardium, and improve cardiac function. P Natl Acad Sci U S A. 2005; 102(10):3766-3771.

16. Orlic D, Kajstura J, Chimenti S, et al. Bone marrow cells regenerate infarcted myocardium. Nature. 2001;410(6829):701-705.

17. Das H, George JC, Joseph M, et al. Stem cell therapy with overexpressed VEGF and PDGF genes improves cardiac function in a rat infarct model. PLoS One. 2009;4(10):e7325.

18. Alvarez-Dolado M, Pardal R, Garcia-Vardugo JM, et al. Fusion of bone-marrow-derived cells with Purkinje neurons, cardiomyocytes and hepatocytes. Nature. 2003;425(6961):968-973.

19. Murry CE, Soonpaa MH, Reinecke H, et al. Haematopoietic stem cells do not transdifferentiate into cardiac myocytes in myocardial infarcts. Nature. 2004;428(6983):664-668.

20. Balsam LB, Wagers AJ, Christensen JL, Kofidis T, Weissman IL, Robbins RC. Haematopoietic stem cells adopt mature haematopoietic fates in ischaemic myocardium. Nature. 2004;428(6983):668-673. 
21. Strauer BE, Brehm M, Zeus T, et al. Repair of infarcted myocardium by autologous intracoronary mononuclear bone marrow cell transplantation in humans. Circulation. 2002;106(15):1913-1918.

22. Dimmeler S, Zeiher AM, Schneider MD. Unchain my heart: the scientific foundations of cardiac repair. J Clin Invest. 2005;115(3): $572-583$.

23. Wollert KC, Meyer GP, Lotz J, et al. Intracoronary autologous bonemarrow cell transfer after myocardial infarction: the BOOST randomised controlled clinical trial. Lancet. 2004;364(9429):141-148.

24. Fernandez-Aviles F, San Roman JA, Garcia-Frade J, et al. Experimental and clinical regenerative capability of human bone marrow cells after myocardial infarction. Circulation Research. 2004;95(7):742-748.

25. de la Fuente LM, Stertzer SH, Argentieri J, et al. Transendocardial autologous bone marrow in chronic myocardial infarction using a helical needle catheter: 1-year follow-up in an open-label, nonrandomized, single-center pilot study (the TABMMI study). American Heart Journal. 2007;154(1):79 e71-e77.

26. Blank U, Karlsson G, Karlsson S. Signaling pathways governing stemcell fate. Blood. 2008;111(2):492-503.

27. Artavanis-Tsakonas S, Rand MD, Lake RJ. Notch signaling: cell fate control and signal integration in development. Science. 1999; 284(5415):770-776.

28. Karanu FN, Murdoch B, Gallacher L, et al. The Notch ligand Jagged-1 represents a novel growth factor of human hematopoietic stem cells. J Exp Med. 2000;192(9):1365-1372.

29. Ohishi K, Varnum-Finney B, Bernstein ID. Delta-1 enhances marrow and thymus repopulating ability of human CD34(+)CD38(-) cord blood cells. J Clin Invest. 2002;110(8):1165-1174.

30. Delaney C, Varnum-Finney B, Aoyama K, Brashem-Stein C, Bernstein ID. Dose-dependent effects of the Notch ligand Delta1 on ex vivo differentiation and in vivo marrow repopulating ability of cord blood cells. Blood. 2005;106(8):2693-2699.

31. Kunisato A, Chiba S, Nakagami-Yamaguchi E, et al. HES-1 preserves purified hematopoietic stem cells ex vivo and accumulates side population cells in vivo. Blood. 2003;101(5):1777-1783.

32. Calvi LM, Adams GB, Weibrecht KW, et al. Osteoblastic cells regulate the haematopoietic stem cell niche. Nature. 2003;425(6960):841-846.

33. Austin TW, Solar GP, Ziegler FC, Liem L, Matthews W. A role for the Wnt gene family in hematopoiesis: Expansion of multilineage progenitor cells. Blood. 1997;89(10):3624-3635.

34. Reya T, Duncan AW, Ailles L, et al. A role for Wnt signalling in self-renewal of haematopoietic stem cells. Nature. 2003;423(6938): 409-414.

35. Blank U, Karlsson G, Moody JL, et al. Smad7 promotes self-renewal of hematopoietic stem cells. Blood. 2006;108(13):4246-4254.

36. Hatzfeld J, Li ML, Brown EL, et al. Release of early human hematopoietic progenitors from quiescence by antisense transforming growth factor beta 1 or Rb oligonucleotides. J Exp Med. 1991;174(4):925-929.

37. Bhatia M, Bonnet $\mathrm{D}, \mathrm{Wu} \mathrm{D}$, et al. Bone morphogenetic proteins regulate the developmental program of human hematopoietic stem cells. J Exp Med. 1999;189(7):1139-1148.

38. Bhardwaj G, Murdoch B, Wu D, et al. Sonic hedgehog induces the proliferation of primitive human hematopoietic cells via BMP regulation. Nat Immunol. 2001;2(2):172-180.

39. Morrison SJ, Spradling AC. Stem cells and niches: mechanisms that promote stem cell maintenance throughout life. Cell. 2008;132(4):598-611.

40. Wilson A, Trumpp A. Bone-marrow haematopoietic-stem-cell niches. Nat Rev Immunol. 2006;6(2):93-106.

41. Osawa M, Hanada K, Hamada H, Nakauchi H. Long-term lymphohematopoietic reconstitution by a single CD34-low/negative hematopoietic stem cell. Science. 1996;273(5272):242-245.

42. Rieger MA, Hoppe PS, Smejkal BM, Eitelhuber AC, Schroeder T. Hematopoietic Cytokines Can Instruct Lineage Choice. Science. 2009; 325(5937):217-218.

43. Taichman RS. Blood and bone: two tissues whose fates are intertwined to create the hematopoietic stem-cell niche. Blood. 2005;105(7): 2631-2639.
44. Wright DE, Bowman EP, Wagers AJ, Butcher EC, Weissman IL. Hematopoietic stem cells are uniquely selective in their migratory response to chemokines. J Exp Med. 2002;195(9):1145-1154.

45. Nagasawa T, Hirota S, Tachibana K, et al. Defects of B-cell lymphopoiesis and bone-marrow myelopoiesis in mice lacking the $\mathrm{CXC}$ chemokine PBSF/SDF-1. Nature. 1996;382(6592):635-638.

46. Distler JH, Jungel A, Kurowska-Stolarska M, et al. Nucleofection: a new, highly efficient transfection method for primary human keratinocytes*. Exp Dermatol. 2005;14(4):315-320.

47. Lataillade JJ, Clay D, Dupuy C, et al. Chemokine SDF-1 enhances circulating CD34(+) cell proliferation in synergy with cytokines: possible role in progenitor survival. Blood. 2000;95(3):756-768.

48. Lee Y, Gotoh A, Kwon HJ, et al. Enhancement of intracellular signaling associated with hematopoietic progenitor cell survival in response to SDF-1/CXCL12 in synergy with other cytokines. Blood. 2002; 99(12):4307-4317.

49. Arai F, Hirao A, Ohmura M, et al. Tie2/angiopoietin-1 signaling regulates hematopoietic stem cell quiescence in the bone marrow niche. Cell. 2004;118(2):149-161.

50. Zhang JW, Niu C, Ye L, et al. Identification of the haematopoietic stem cell niche and control of the niche size. Nature. 2003;425(6960): 836-841.

51. Maeno M, Mead PE, Kelley C, et al. The role of BMP-4 and GATA2 in the induction and differentiation of hematopoietic mesoderm in Xenopus laevis. Blood. 1996;88(6):1965-1972.

52. Hirsch E, Iglesias A, Potocnik AJ, Hartmann U, Fassler R. Impaired migration but not differentiation of haematopoietic stem cells in the absence of beta1 integrins. Nature. 1996;380(6570):171-175.

53. Levesque JP, Simmons PJ. Cytoskeleton and integrin-mediated adhesion signaling in human $\mathrm{CD}^{+} 4^{+}$hemopoietic progenitor cells. Exp Hematol. 1999;27(4):579-586.

54. Higuchi A, Yang ST, Li PT, et al. Polymeric Materials for Ex vivo Expansion of Hematopoietic Progenitor and Stem Cells. Polym Rev. 2009;49(3):181-200.

55. Ingber D. Extracellular matrix and cell shape: potential control points for inhibition of angiogenesis. J Cell Biochem. 1991;47(3):236-241.

56. Chua KN, Chai C, Lee PC, Ramakrishna S, Leong KW, Mao HQ. Functional nanofiber scaffolds with different spacers modulate adhesion and expansion of cryopreserved umbilical cord blood hematopoietic stem/progenitor cells. Exp Hematol. 2007;35(5):771-781.

57. McBeath R, Pirone DM, Nelson CM, Bhadriraju K, Chen CS. Cell shape, cytoskeletal tension, and RhoA regulate stem cell lineage commitment. Dev Cell. 2004;6(4):483-495.

58. Guilak F, Cohen DM, Estes BT, Gimble JM, Liedtke W, Chen CS. Control of stem cell fate by physical interactions with the extracellular matrix. Cell Stem Cell. 2009;5(1):17-26.

59. Engler AJ, Sen S, Sweeney HL, Discher DE. Matrix elasticity directs stem cell lineage specification. Cell. 2006;126(4):677-689.

60. Winer JP, Janmey PA, McCormick ME, Funaki M. Bone marrowderived human mesenchymal stem cells become quiescent on soft substrates but remain responsive to chemical or mechanical stimuli. Tissue Eng Part A. 2009;15(1):147-154.

61. Saha K, Keung AJ, Irwin EF, et al. Substrate modulus directs neural stem cell behavior. Biophys J. 2008;95(9):4426-4438.

62. Christopherson GT, Song H, Mao HQ. The influence of fiber diameter of electrospun substrates on neural stem cell differentiation and proliferation. Biomaterials. 2009;30(4):556-564.

63. Yim EKF, Pang SW, Leong KW. Synthetic nanostructures inducing differentiation of human mesenchymal stem cells into neuronal lineage. Exp Cell Res. 2007;313(9):1820-1829.

64. Arnold M, Cavalcanti-Adam EA, Glass R, et al. Activation of integrin function by nanopatterned adhesive interfaces. Chemphyschem. 2004; 5(3):383-388.

65. Kantawong F, Burgess KE, Jayawardena K, et al. Whole proteome analysis of osteoprogenitor differentiation induced by disordered nanotopography and mediated by ERK signalling. Biomaterials. 2009; 30(27):4723-4731. 
66. Martin MJ, Muotri A, Gage F, Varki A. Human embryonic stem cells express an immunogenic nonhuman sialic acid. Nat Med. 2005; 11(2):228-232.

67. Douay L. Experimental culture conditions are critical for ex vivo expansion of hematopoietic cells. J Hematoth Stem Cell. 2001; 10(3):341-346.

68. Yoshida T, Hazan I, Zhang J, et al. The role of the chromatin remodeler Mi-2 beta in hematopoietic stem cell self-renewal and multilineage differentiation. Gene Dev. 2008;22(9):1174-1189.

69. Araki H, Yoshinaga K, Boccuni P, Zhao Y, Hoffman R, Mahmud N. Chromatin-modifying agents permit human hematopoietic stem cells to undergo multiple cell divisions while retaining their repopulating potential. Blood. 2007;109(8):3570-3578.

70. Fraser CC, Eaves CJ, Szilvassy SJ, Humphries RK. Expansion In vitro of Retrovirally Marked Totipotent Hematopoietic Stem-Cells. Blood. 1990;76(6):1071-1076.

71. Miller CL, Eaves CJ. Expansion in vitro of adult murine hematopoietic stem cells with transplantable lympho-myeloid reconstituting ability. Proc Natl Acad Sci U S A. 1997;94(25):13648-13653.

72. Iscove NN, Nawa K. Hematopoietic stem cells expand during serial transplantation in vivo without apparent exhaustion. Curr Biol. 1997; 7(10):805-808.

73. Ueda T, Tsuji K, Yoshino H, et al. Expansion of human NOD/SCIDrepopulating cells by stem cell factor, Flk2/Flt3 ligand, thrombopoietin, IL-6, and soluble IL-6 receptor. J Clin Invest. 2000;105(7): 1013-1021.

74. Bhatia M, Bonnet D, Kapp U, Wang JC, Murdoch B, Dick JE. Quantitative analysis reveals expansion of human hematopoietic repopulating cells after short-term ex vivo culture. J Exp Med. 1997;186(4): 619-624.

75. Glimm H, Eaves CJ. Direct evidence for multiple self-renewal divisions of human in vivo repopulating hematopoietic cells in short-term culture. Blood. 1999;94(7):2161-2168.

76. Borge OJ, Ramsfjell V, Veiby OP, Murphy MJ Jr, Lok S, Jacobsen SE. Thrombopoietin, but not erythropoietin promotes viability and inhibits apoptosis of multipotent murine hematopoietic progenitor cells in vitro. Blood. 1996;88(8):2859-2870.

77. Cashman JD, Eaves CJ. Human growth factor-enhanced regeneration of transplantable human hematopoietic stem cells in nonobese diabetic/ severe combined immunodeficient mice. Blood. 1999;93(2):481-487.

78. Bagley J, Rosenzweig M, Marks DF, Pykett MJ. Extended culture of multipotent hematopoietic progenitors without cytokine augmentation in a novel three-dimensional device. Exp Hematol. 1999; 27(3):496-504.
79. LaIuppa JA, McAdams TA, Papoutsakis ET, Miller WM. Culture materials affect ex vivo expansion of hematopoietic progenitor cells. J Biomed Mater Res. 1997;36(3):347-359.

80. Franke K, Pompe T, Bornhauser M, Werner C. Engineered matrix coatings to modulate the adhesion of $\mathrm{CD} 133^{+}$human hematopoietic progenitor cells. Biomaterials. 2007;28(5):836-843.

81. Sagar BM, Rentala S, Gopal PN, Sharma S, Mukhopadhyay A. Fibronectin and laminin enhance engraftibility of cultured hematopoietic stem cells. Biochem Biophys Res Commun. 2006;350(4):1000-1005.

82. Jiang XS, Chai C, Zhang Y, Zhuo RX, Mao HQ, Leong KW. Surfaceimmobilization of adhesion peptides on substrate for ex vivo expansion of cryopreserved umbilical cord blood CD34(+) cells. Biomaterials. 2006;27(13):2723-2732.

83. Chua KN, Chai C, Lee PC, et al. Surface-aminated electrospun nanofibers enhance adhesion and expansion of human umbilical cord blood hematopoietic stem/progenitor cells. Biomaterials. 2006;27(36): 6043-6051.

84. Feng Q, Chai C, Jiang XS, Leong KW, Mao HQ. Expansion of engrafting human hematopoietic stem/progenitor cells in three-dimensional scaffolds with surface-immobilized fibronectin. J Biomed Mater Res A. 2006;78(4):781-791.

85. Das H, Abdulhameed N, Joseph M, Sakthivel R, Mao HQ, Pompili VJ. Ex vivo nanofiber expansion and genetic modification of human cord blood-derived progenitor/stem cells enhances vasculogenesis. Cell Transplant. 2009;18(3):305-318.

86. Templin C, Kotlarz D, Faulhaber J, et al. Ex vivo expanded hematopoietic progenitor cells improve cardiac function after myocardial infarction: role of beta-catenin transduction and cell dose. $\mathrm{J} \mathrm{Mol} \mathrm{Cell}$ Cardiol. 2008;45(3):394-403.

87. Hai-Jiang W, Xin-Na D, Hui-Jun D. Expansion of hematopoietic stem/progenitor cells. Am J Hematol. 2008;83(12):922-926.

88. Smith RR, Barile L, Messina E, Marban E. Stem cells in the heart: What's the buzz all about? Part 2: Arrhythmic risks and clinical studies. Heart Rhythm. 2008;5(6):880-887.

89. George JC, Goldberg J, Joseph M, et al. Transvenous intramyocardial cellular delivery increases retention in comparison to intracoronary delivery in a porcine model of acute myocardial infarction. $J$ Interv Cardiol. 2008;21(5):424-431.

90. Sanchez PL, Villa A, Sanz R, et al. Present and future of stem cells for cardiovascular therapy. Ann Med. 2007;39(6):412-427.

91. Mathur A, Martin JF. Stem cells and repair of the heart. Lancet. 2004; 364(9429):183-192.

92. Wollert KC, Drexler H. Clinical applications of stem cells for the heart. Circulation Research. 2005;96(2):151-163.
Stem Cells and Cloning: Advances and Applications

\section{Publish your work in this journal}

Stem Cells and Cloning: Advances and Applications is an international, peer-reviewed, open access journal. Areas of interest in stem cell research include: Embryonic cell stems; Adult stem cells; Blastocysts; Cordblood stem cells; Stem cell transformation and culture; Therapeutic cloning; Umbilical cord blood and bone marrow cells; Laboratory,

\section{Dovepress}

animal and human therapeutic studies; Philosophical and ethical issues related to stem cell research. This journal is indexed on CAS. The manuscript management system is completely online and includes a quick and fair peer-review system. Visit http://www.dovepress.com/ testimonials.php to read real quotes from published authors. 